Supporting Information for

\title{
Heterogeneity of Water Molecules on the Free Surface of Thin Reduced
}

\section{Graphene Oxide Sheets}

Zhuo Liu ${ }^{1,2,4, \ddagger}$, Chenxing Yang ${ }^{1,2, \ddagger}$, Lei Zhang, ${ }^{2,3, \ddagger}$, Yuanxi Yu ${ }^{1}$, Minhao Yu ${ }^{1,2}$, Victoria García

Sakai $^{5}$, Madhusudan Tygai ${ }^{6,7}$, Takeshi Yamada ${ }^{8}$, Lunhua $\mathrm{He}^{9,10}$, Xiaohua Zhang ${ }^{11,12,13}$, and Liang Hong ${ }^{*, 1,2}$

${ }^{1}$ School of Physics and Astronomy, ${ }^{2}$ Institute of Natural Sciences, and ${ }^{3}$ School of Materials Science and Engineering, Shanghai Jiao Tong University, Shanghai 200240, P. R. China ${ }^{4}$ Institute for Advanced Study, The Hong Kong University of Science and Technology, Hong Kong, China

${ }^{5}$ ISIS Neutron and Muon Facility, Rutherford Appleton Laboratory, Science \& Technology Facilities Council, Didcot OX11 0QX, United Kingdom

${ }^{6}$ National Institute of Standards and Technology (NIST), NIST Center for Neutron Research, Gaithersburg, Maryland 20899, United States

${ }^{7}$ Department of Materials Science and Engineering, University of Maryland, College Park, Maryland 20742, United States

${ }^{8}$ Neutron Science and Technology Center, Comprehensive Research Organization for Science and Society, 162-1 Shirakata, Tokai, Naka, Ibaraki 319-1106, Japan

${ }^{9}$ Beijing National Laboratory for Condensed Matter Physics, Institute of Physics, Chinese Academy of Sciences, Beijing 100190, China

${ }^{10}$ Spallation Neutron Source Science Center, Dongguan 523803, China 
${ }^{11}$ Center for Soft Condensed Matter Physics and Interdisciplinary Research, ${ }^{12}$ School of Physical Science and Technology and ${ }^{13}$ Jiangsu Key Laboratory of Thin Films, Soochow University, Suzhou 215006, China

These authors contributed equally to this work.

*e-mail: hong13liang@sjtu.edu.cn 
Thermogravimetric Analysis (TGA). The water content of rGO after the heating process was measured using TGA at a heating rate of $5 \mathrm{~K} / \mathrm{min}$ from $25^{\circ} \mathrm{C}$ to $110^{\circ} \mathrm{C}$ with nitrogen purged. The measured weight loss is assumed to arise entirely from evaporation of water from the sample. Figure $\mathrm{S} 1$ shows the TGA curves for the rGO samples after the heating process, which is $\sim 0.4 \%$ by weight loss.

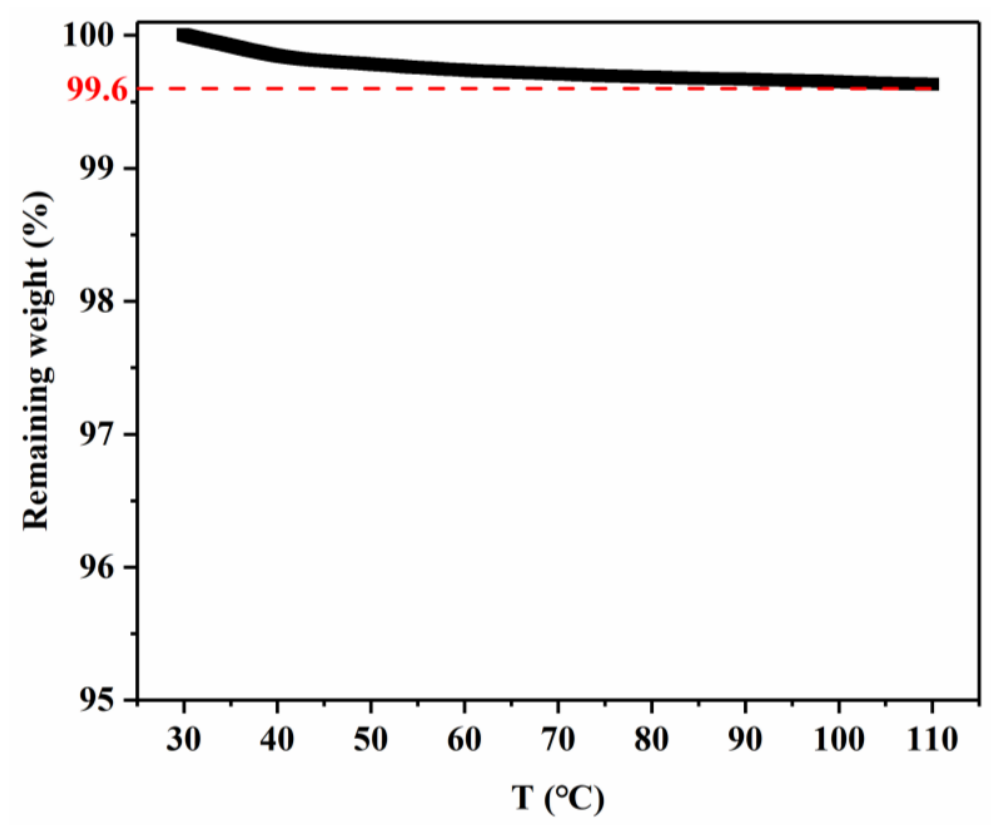

Figure S1. Thermogravimetric analysis of water content in the dehydrated rGO powder. The red dash line shows that the remaining weight is $99.6 \%$ at $110{ }^{\circ} \mathrm{C}$, suggesting the hydration level of dehydrated rGO is $0.4 \%$, negligibly small. 
X-ray photoelectron spectroscopy (XPS). The C/O atom ratio of our rGO sample is 90:10. As shown in Figure S2, the XPS spectrum of rGO was deconvoluted (CasaXPS software) into three peaks corresponding to: carbon $\mathrm{sp}^{2}$ and $\mathrm{sp}^{3}(\mathrm{C})$, epoxy and hydroxyls $(\mathrm{C}-\mathrm{O})$, and carboxylates $(\mathrm{O}-\mathrm{C}=\mathrm{O}) .{ }^{1}$ As a rough estimate, the sample consists of $\sim 70 \%$ epoxy and hydroxyls, and $\sim 30 \%$ carboxylates.

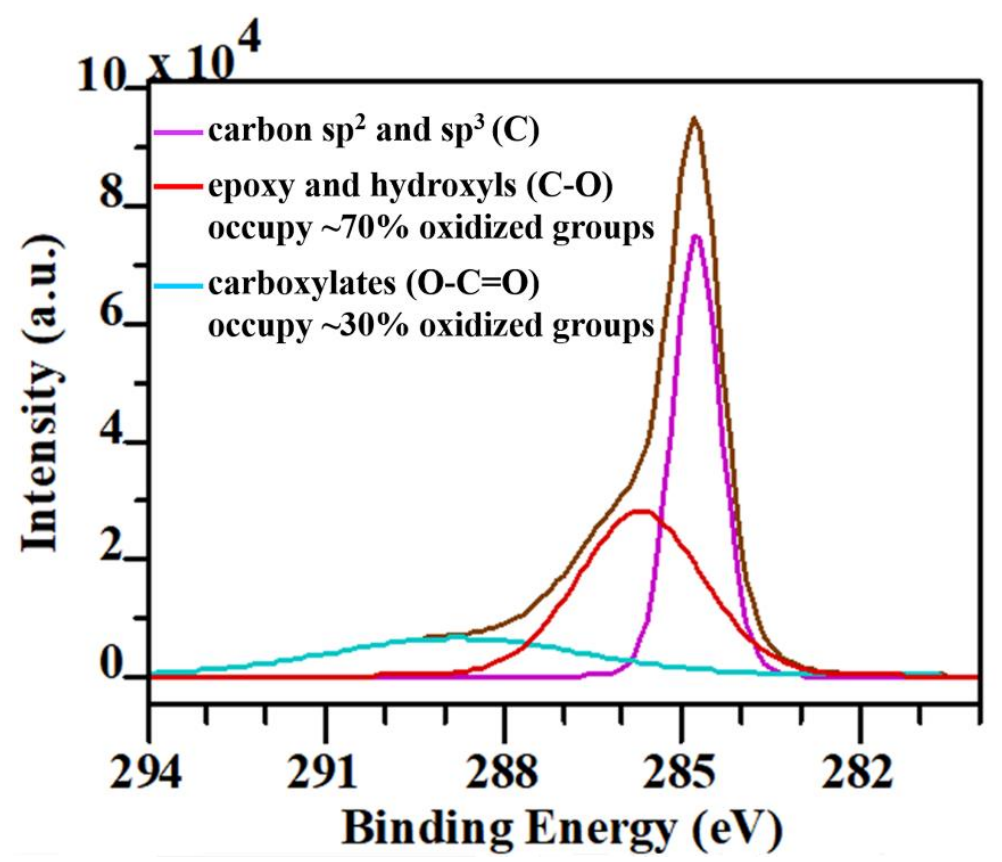

Figure S2. XPS analysis on rGO sample. The XPS spectrum (brown line) of the rGO sample and its deconvolution as carbon $\mathrm{sp}^{2}$ and $\mathrm{sp}^{3}$ (purple), epoxy and hydroxyls (red) and carboxylates (cyan) functional groups. 
Neutron Diffraction (ND). The neutron diffraction patterns of $\mathrm{rGO}+(0.1) \mathrm{D}_{2} \mathrm{O}$ and $\mathrm{rGO}+(0.6) \mathrm{D}_{2} \mathrm{O}$ in the $d$ spacing range from 1.5 to $5 \AA$ were collected at $180 \mathrm{~K}$ at General Purpose Powder Diffraction (GPPD) at the China Spallation Neutron Source (CSNS) to identify the ice structure formed on rGO in a wide $d$ spacing range (see Figure S3). The peaks of $\mathrm{rGO}+(0.10) \mathrm{D}_{2} \mathrm{O}$ at 2.23 and $3.66 \AA$ can be attributed to the diffraction from $\mathrm{rGO}, 2,3$ suggesting no ice forming at the hydration level of 0.10 at $180 \mathrm{~K}$. In contrast, the extra peaks appear on $\mathrm{rGO}+(0.58) \mathrm{D}_{2} \mathrm{O}$ at 3.90, 3.67, 3.45, 2.67 and $2.14 \AA$ (Figure $\mathrm{S} 3$ ), which correspond to the (100), (002), (101), (102) and (103) planes of hexagonal ice. ${ }^{4}$

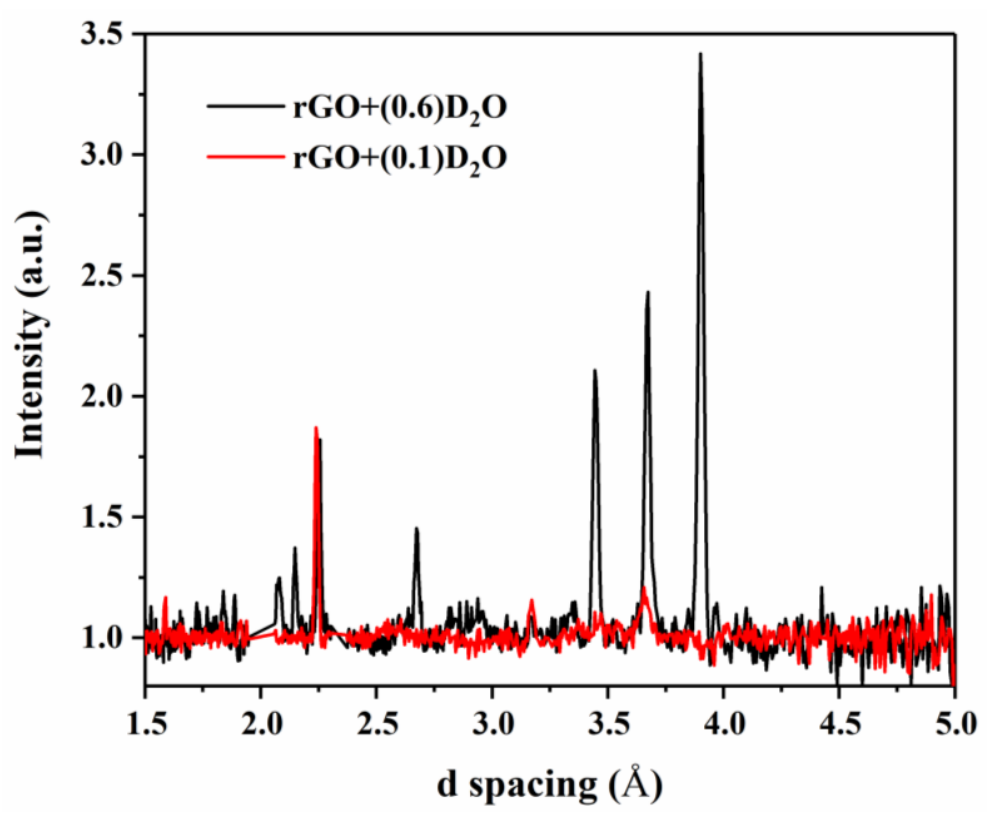

Figure S3. Neutron diffraction of water on rGO. The neutron diffraction patterns of $\mathrm{rGO}+(0.1) \mathrm{D}_{2} \mathrm{O}($ red $)$ and $\mathrm{rGO}+(0.6) \mathrm{D}_{2} \mathrm{O}$ (black). 


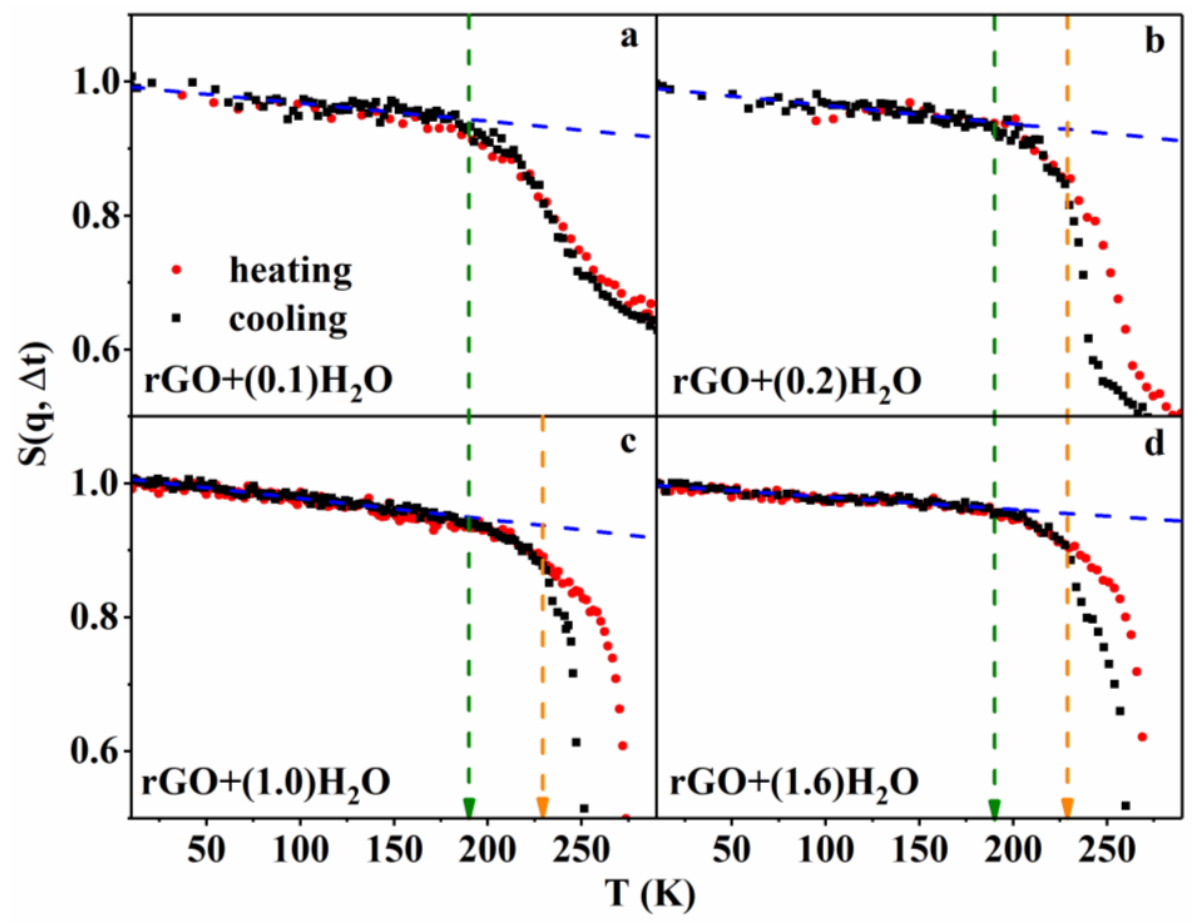

Figure S4. The existence of non-freezing bound water on rGO at all hydration levels. a-

d, Experimental $S(\boldsymbol{q}, \Delta t)$ for $(\mathbf{a}) \mathrm{rGO}+(0.1) \mathrm{H}_{2} \mathrm{O}$, (b) $\mathrm{rGO}+(0.2) \mathrm{H}_{2} \mathrm{O},(\mathbf{c}) \mathrm{rGO}+(1.0) \mathrm{H}_{2} \mathrm{O}$ and (d) $\mathrm{rGO}+(1.6) \mathrm{H}_{2} \mathrm{O}$ during both cooling (red circles) and heating (black squares). Here, $\Delta \boldsymbol{t}=1$ $n s$. The $S(\boldsymbol{q}, \Delta t)$ in the temperature range from $10 \mathrm{~K}$ to $290 \mathrm{~K}$ are shown. The blue dash lines are the linear fits of $S(\boldsymbol{q}, \Delta t)$ during cooling in the temperature range from $10 \mathrm{~K}$ to $170 \mathrm{~K}$, and the green arrows mark the temperature that $S(\boldsymbol{q}, \Delta t)$ bends downwards at $\sim 190 \mathrm{~K}$ at each subfigure, suggesting the existence of non-freezing bound water on rGO at all hydration levels. The low-temperature freezing water appears around $190 \mathrm{~K}$ as the beginning of the hysteresis, and it is clearly separated from the second freezing step around $230 \mathrm{~K}$ in the cooling cycle by a plateau region (see the orange arrows). 


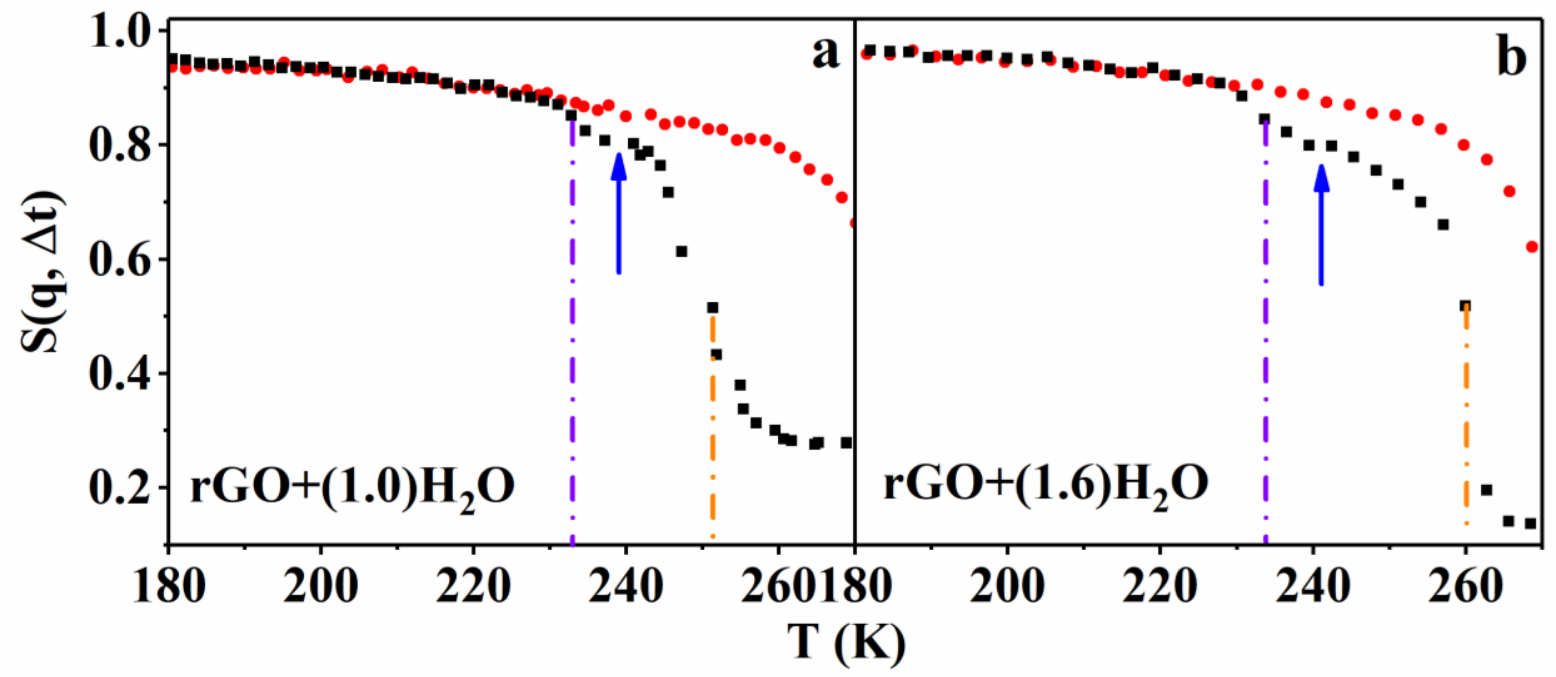

Figure S5. The existence of freezing bound water on rGO at the hydration levels of 1.0 and 1.6. a-b, Experimental $S(\boldsymbol{q}, \Delta t)$ for (a) $\mathrm{rGO}+(1.0) \mathrm{H}_{2} \mathrm{O}$ and (b) $\mathrm{rGO}+(1.6) \mathrm{H}_{2} \mathrm{O}$ during both heating (red circles) and cooling (black squares). Here, $\Delta t=1 n s$. For clarity, only the $S(\boldsymbol{q}, \Delta t)$ in the temperature range from $180 \mathrm{~K}$ to $270 \mathrm{~K}$ are shown. The purple and orange dash dot lines mark the ice forming temperatures during the cooling process, defined as the inflection point within the freezing step. The blue arrows mark the separation between the two ice-forming events. 


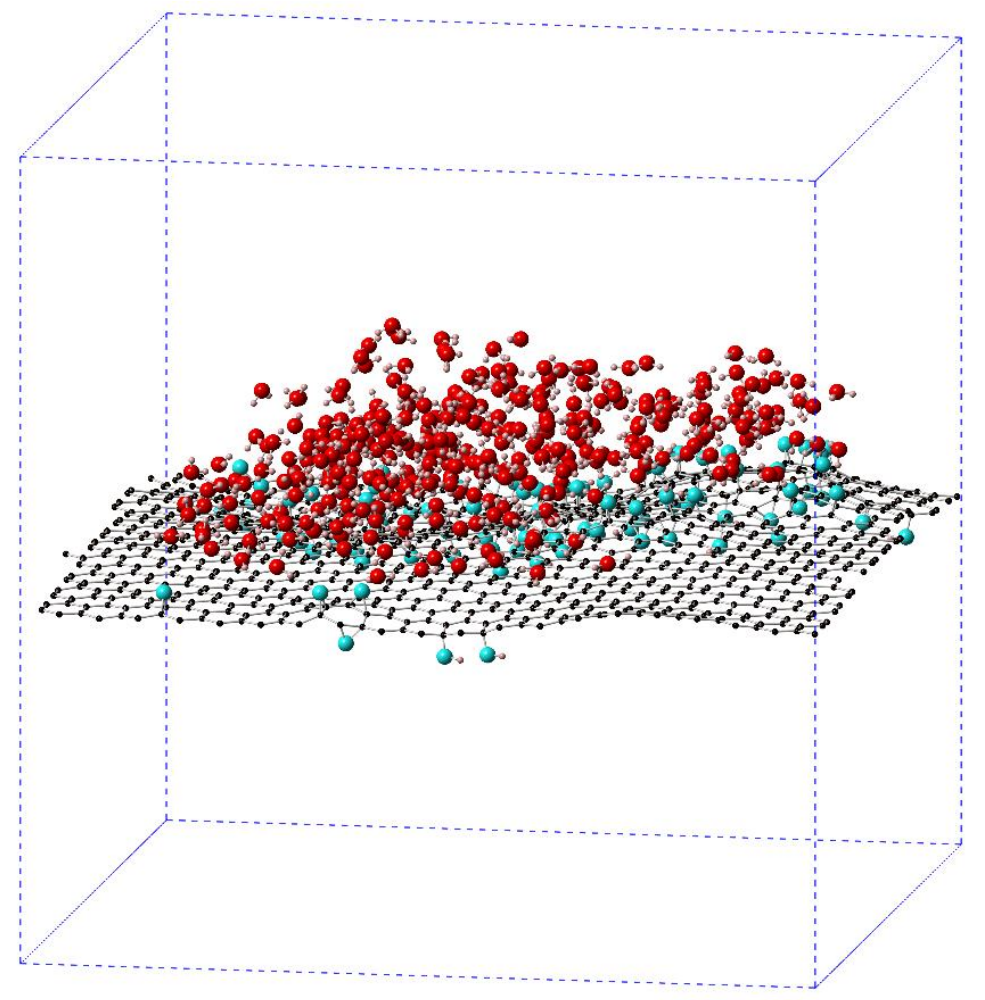

Figure S6. The MD simulation box of $\mathrm{H}_{2} \mathrm{O}$ on $\mathrm{rGO}$ at $\boldsymbol{h}=\mathbf{1 . 0}$. White, black, cyan and red spheres represent hydrogen atoms, carbon atoms, oxygen atoms of oxide groups and water, respectively. The blue dash cubic represents the simulation box. 

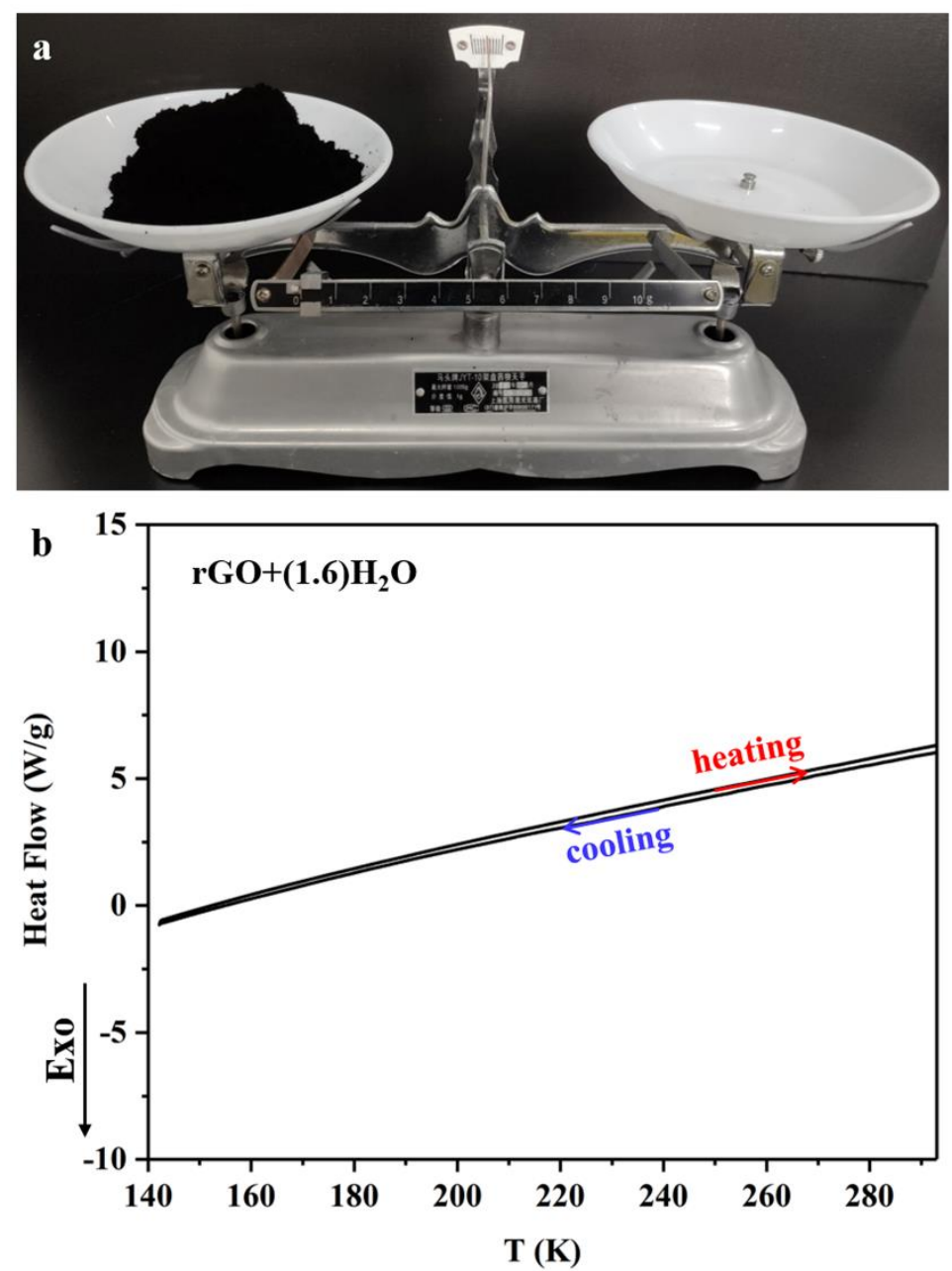

Figure S7. The ultra-low density rGO powder $\left(\sim 0.005 \mathrm{~g} / \mathrm{cm}^{3}\right)$ and its differential scanning calorimetry (DSC) measurement at $\boldsymbol{h}=\mathbf{1 . 6}$. a, Comparison of volumes of 1 gram rGO powder in the left pan with 1 gram load in the right pan on a balance. $\mathbf{b}$, Heat flow of the rGO powder at $h=1.6$ at a heating and cooling rate of $5 \mathrm{~K} / \mathrm{min}$ measured by the differential scanning calorimeter (DSC 204F1, Netzsch). No noticeable endothermic and exothermic peaks, characteristic for phase transition, are detected as the sample density is too low. 


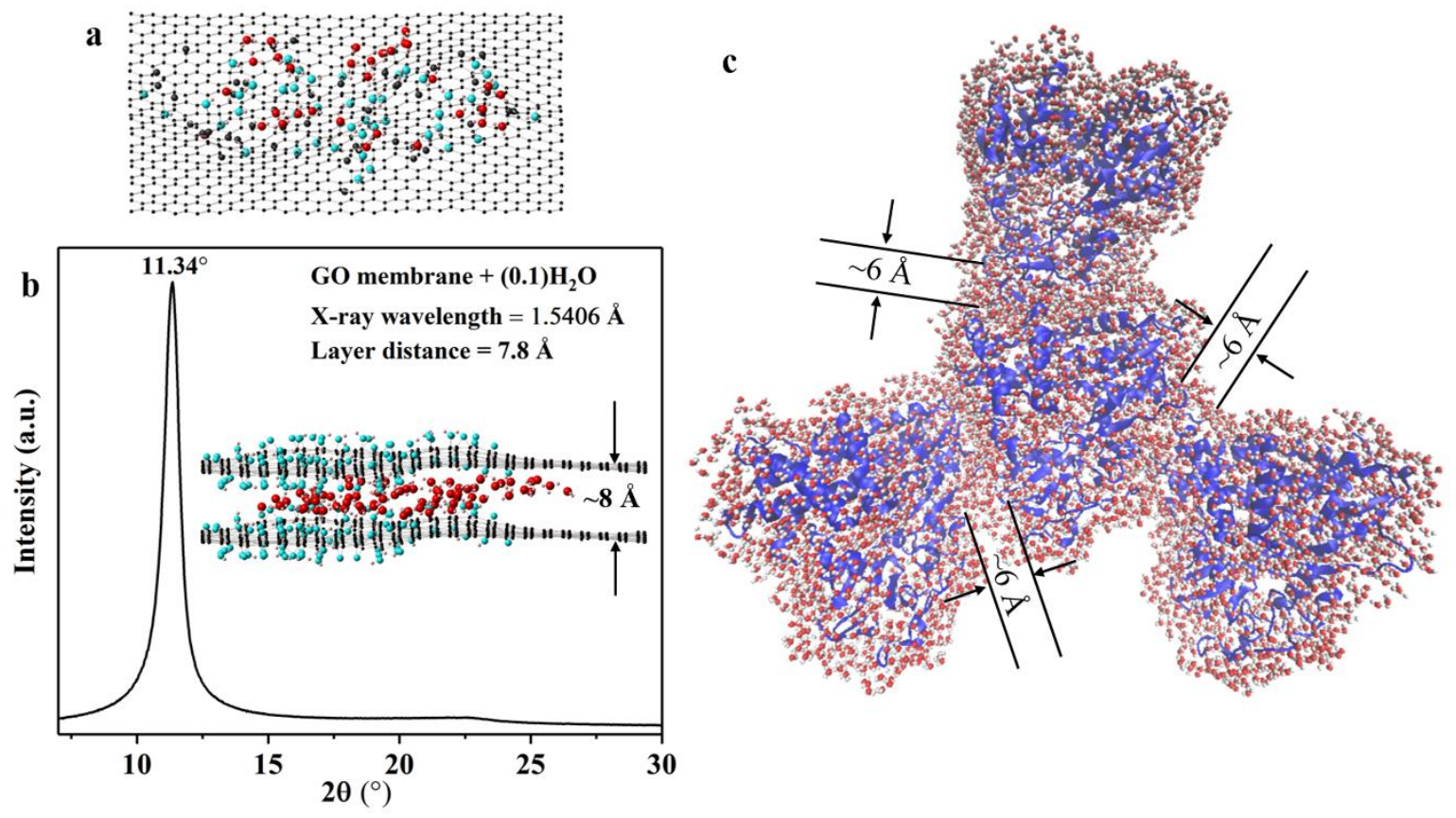

Figure S8. Schematic view of non-freezing bound water. a, Water on rGO with $h=0.1$. b, The Powder X-ray diffraction pattern of GOM at $h=0.1$ with the interlayer distance of $\sim 7.8$ $\AA$, and the inset is the schematic presentation of bound water inside GOM. c, Water between protein molecules (X-ray crystal structure, PDB: 2ZAX) with inter-protein distance of $\sim 6 \AA$. White, black, cyan and red spheres represent hydrogen atoms, carbon atoms, oxygen atoms of oxide groups and water, respectively. The blue cartoons represent protein molecules. 


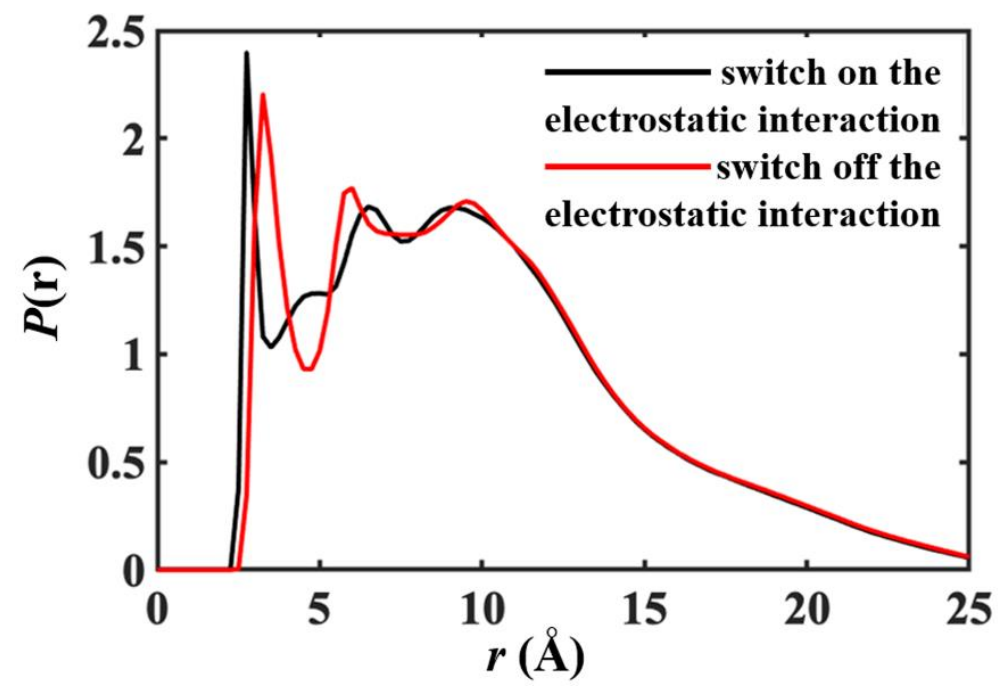

Figure S9. MD-derived Spatial distribution of water with and without the electrostatic interaction with oxide groups on monolayer rGO. The probability distributions, $P(r)$, of water molecules as a function of distance, $r$, from the nearest oxide group on rGO at $h=3.0$ with (black) and without (red) the electrostatic interaction. As seen in this figure, the first hydration layer, determined by the first minimum of $P(r)$, broadens from 3 to $5 \AA$ when switching off the electronic charge of atoms in rGO as the oxide groups exert much less energetic constrain on the surrounding water molecules. 


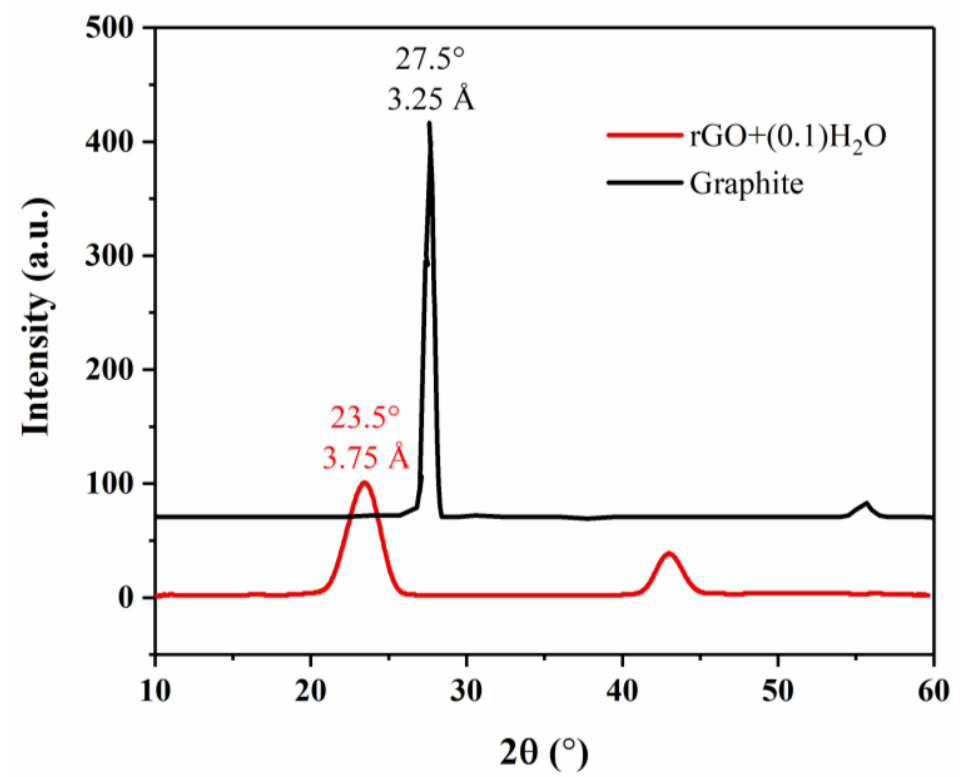

Figure S10. The powder X-ray diffraction patterns of $\mathrm{rGO}+(0.1) \mathrm{H}_{2} \mathrm{O}$ (red) and graphite (black). The PXRD data of graphite are extracted from Fig. 4 in Ref. 5. 


\section{References}

(1) Yumitori, S. Correlation of c1s chemical state intensities with the o1s intensity in the xps analysis of anodically oxidized glass-like carbon samples. J. Mater. Sci. 2000, 35, 139-146.

(2) Tang, L.; Wang, Y.; Li, Y.; Feng, H.; Lu, J.; Li, J. Preparation, structure, and electrochemical properties of reduced graphene sheet films. Adv. Funct. Mater. 2009, 19, 2782-2789.

(3) Stobinski, L.; Lesiak, B.; Malolepszy, A.; Mazurkiewicz, M.; Mierzwa, B.; Zemek, J.; Jiricek, P.; Bieloshapka, I. Graphene oxide and reduced graphene oxide studied by the xrd, tem and electron spectroscopy methods. J. Electron. Spectrosc. Relat. Phenom. 2014, 195, 145-154.

(4) Kolesnikov, A. I.; Sinitsyn, V. V.; Ponyatovsky, E. G.; Natkaniec, I.; Smirnov, L. S.; Li, J. C. Neutron-scattering studies of ice prepared by different thermobaric treatments. J. Phys. Chem. B 1997, 101, 6082-6086.

(5) Chen, Y.; Niu, Y.; Tian, T.; Zhang, J.; Wang, Y.; Li, Y.; Qin, L.-C. Microbial reduction of graphene oxide by azotobacter chroococcum. Chem. Phys. Lett. 2017, 677, 143-147. 